\title{
Desempeño del personal del área de farmacia de la Clínica Médica san Juan de Dios ${ }^{1}$
}

\author{
Aura Dilsia Moreno Torrez ${ }^{2}$ \\ Elba Lisbeth Ruiz Hernàndez ${ }^{3}$ \\ Yasari Lisbeth Pérez Pérez ${ }^{4}$ \\ Beverly Castillo Herrera ${ }^{5}$
}

\section{RESUMEN}

En esta investigación se detalla el diseño del parámetro de evaluación que utilizan los jefes para medir el desempeño de cada trabajador/a, así mismo la opinión de los/as asegurados en cuanto a la atención que reciben de esta importante área, el análisis de las fortalezas, oportunidades debilidades y amenazas (FODA), permitió proponer alternativas que pueden ser implementadas para una mayor eficiencia. Esta es una investigación cualitativa. Se aplicaron entrevistas a directores y trabajadores, y se aplicó una encuesta a los usuarios. Entre los resultados se muestra que los/as trabajadores/as presentan un déficit en su desempeño laboral, se planifica incorrectamente, los objetivos institucionales no están claros, existe un clima desagradable de trabajo: negatividad, egoísmo, desmotivación, y desconfianza. Esta situación repercute en la atención a los usuarios. Se recomienda la creación de estándares de servicio para una atención a sus clientes, capacitación a empleados, y crear un sistema formal de quejas y sugerencias para conocer la opinión del usuario.

Palabras Claves: Evaluación, Desempeño, Trabajadores, Usuarios

Recibido: 15 de diciembre de 2014

Aceptado: 22 de junio de 2015

1 Este artículo se basa en la investigación titulada Desempeño del personal del área de farmacia de la clínica médica san juan de Dios. Para obtener el título de Licenciado en Administración de Empresas de la UNAN - Managua, FAREM - Estelí.

2 UNAN - Managua, FAREM - Estelí, Correo electrónico: aura.moreno93@yahoo.es

3 UNAN - Managua, FAREM - Estelí, Correo electrónico: elbaruiz07@gmail.com

4 UNAN - Managua, FAREM - Estelí, Correo electrónico: yasari.perez@yahoo.es

5 Candidata a doctora en Ciencias Sociales por la Universidad del Zulia. Maestría en Ciencias Sociales por la Universidad de Guadalajara. Docente titular de la UNAN-Managua, FAREM-Estelí. Correo Electrónico:

beverly.castillo@yahoo.com. 


\title{
Staff Performance in the pharmacy at Medical Clinic San Juan de Dios
}

\begin{abstract}
In this research design evaluation parameters used by bosses to measure the performance of each worker is detailed, also the opinion of the insurance workers as to the assistance they receive from this important area, the analysis of the strengths, weaknesses, opportunities, and threats (FODA), allowed to propose alternatives that can be implemented for greater efficiency. This is a qualitative research. Interviews with managers and workers were applied, and a survey to users. The results show that the workers present a deficit in their job performance, it is planned incorrectly, institutional objectives are not clear, there is an unpleasant working environment: negativity, selfishness, lack of motivation, and distrust. This situation affects the service to users. The establishment of service standards for customer care and employee training is recommended, and the creation of a formal system of complaints and suggestions to get feedback from the user.
\end{abstract}

Keywords: Evaluation, Performance, Employees, Users 


\section{INTRODUCCIÓN}

En la actualidad se reconoce que las personas constituyen el pilar fundamental de toda empresa; el éxito o fracaso de la misma depende del trabajo efectuado por su personal. Los administradores deben prestar especial atención a este recurso tan imprescindible e importante para la buena atención de los asegurados.

El nivel de resolución que ha alcanzado la Clínica San Juan de Dios, implica una serie de procesos necesarios para alcanzar la mejor calidad en pos de la atención del asegurado, lo que significa un incremento en los recursos humanos y técnicos, así como también fortalecer el sistema contable de la Clínica para obtener resultados contables confiables, creando un plan de mejoramiento continuo de la calidad que sea medible y alcanzable.

Según el Dr. José Ignacio Rosales, Director general de la clínica previsional, considera que la situación que enfrenta el área de farmacia afecta directamente a dicha institución, presentando déficit en cuanto a las atenciones que brindan sustentado en las quejas que los asegurados realizan constantemente. Se presume que dentro de sus causas figuran; la falta de trabajo en equipo, mala comunicación del grupo, falta de disponibilidad del personal, que fomenta desmotivación e insuficiencia para realizar las funciones asignadas, ausencia de subordinación, llegadas tardes al trabajo, esto refleja falta de ética profesional, desorganización laboral y no desempeñarse con éxito en su área de trabajo. Otro elemento que influye son los factores externos al lugar de trabajo, que pueden ocasionar frustración, y tensión en el personal.

El crecimiento de la clínica tanto en equipos médicos y atención a sus asegurados es notorio, pero la problemática de esta área está afectando indirectamente su desarrollo, provocando quejas e insatisfacción en los pacientes que son el recurso más importante para la sostenibilidad de la clínica. El presente trabajo tiene como objetivo proponer estrategias que contribuyan al eficiente desempeño laboral en el área de farmacia de la Clínica Médica Previsional MINSA Filial San Juan de Dios de la ciudad de Estelí 2013 - 2014, laLos resultados de la investigación van a ser relevantes para enriquecer el conocimiento y a la vez, servir como fuente de información para futuras investigaciones, sobre el desempeño laboral de recursos humanos.

\section{MATERIALES Y MÉTODOS}

Para llevar a cabo el estudio se utilizaron fuentes primarias de carácter cualitativo, cuyos instrumentos fueron una guía de entrevista aplicada a informantes claves, se obtuvo información acerca del desempeño laboral según el criterio cada trabajador y las estrategias que podrían implementarse para mejorar el rendimiento laboral, una guía de observación en el área de estudio para observar el desempeño del personal, una encuesta aplicada a 212 usuarios asegurados que hayan solicitado al menos dos veces el servicio, para conocer la valoración del desempeño del personal por parte de los usuarios. También se hizo una revisión bibliográfica de diversas fuentes como internet, biblioteca urania Zelaya Farem-Esteli, e información brindada por el área de recursos humanos de la clínica.

\section{RESULTADOS}

Para tener un soporte teórico, se elaboró el marco teórico-conceptual de investigación en base a: Empresa, recursos humanos y evaluación del desempeño. La empresa "es una organización social que utiliza una gran variedad de recursos para alcanzar determinados objetivos". Explicando este concepto, el autor menciona que la empresa "es una organización social por ser una asociación de personas para la explotación de un negocio y que tiene por fin un determinado objetivo, que puede ser el lucro o la atención de una necesidad social”. (Chiavenato, 1994, pág. 4). 
El estudio de las personas constituye la unidad básica de las organizaciones, especialmente en la administración de los recursos humanos. El área de recursos humanos tiene diversas vertientes para estudiar: las personas como personas (dotadas de características propias, de personalidad e individualidad, aspiraciones, valores, actitudes, motivación y objetivos individuales); y a las personas como recursos (dotadas de habilidades, capacidades y conocimientos necesarios para la tarea organizacional. (Chiavenato 1994, pág. 163).

Evaluación del desempeño es una técnica de dirección imprescindible en la actividad administrativa. Es el procedimiento para evaluar el personal se denomina evaluación de desempeño y generalmente, se elabora a partir de programas formales de evaluación, basados en una cantidad razonable de informaciones respecto a los empleados y a su desempeño en el cargo. Su función es estimular o buscar el valor, la excelencia y las cualidades de alguna persona. Medir el desempeño del individuo en el cargo y de su potencial de desarrollo. (Butteriss, 2000, pág. 56).

\section{Descripción General de las clínicas médicas previsionales en Nicaragua}

Las Clínicas Médicas Previsionales surgieron en 1997, con las características servicio de pensionados en los Hospitales, posteriormente evolucionaron a las Empresa Medicas Previsionales del MINSA y el $1^{\circ}$ de Octubre del 2007 con el Gobierno de Reconciliación Nacional a través del Ministerio de Salud se crean las Clínicas Médicas Previsionales del MINSA, que nace como proyecto del Gobierno con el fin de brindarles mejores servicios médicos a los trabajadores asegurados de Nicaragua. En todas estas distintas modalidades de atención en salud se han aplicado todos los procedimientos de contabilidad previamente aceptados, al igual que las normas nacionales dictadas por la ley de contrataciones del estado y las normas de control interno de la Contraloría General de la República.
El funcionamiento de estas Clínicas está determinado por las políticas establecidas por el MINSA y las regulaciones particulares del INSS, ya que este último es el que establece los alcances de las atenciones médicas, es decir establece las lista de prestaciones de servicios de salud y lista de medicamentos autorizadas y convenidas con la clínica médica previsional para brindarles a los derecho habientes afiliados a la Clínica; igualmente supervisan la Calidad de la atención que brindan las Clínicas, es parte integrante de la Corporación de Clínicas Médicas de nuestro país.

Actualmente se atiende un segmento de la población asegurada del país clasificada en cotizantes de régimen integral, riesgo laboral (accidentes de trabajo), adultos mayores (jubilados, pensión de vejez) y plan facultativo.

\section{Caracterización de la clínica médica previsional MINSA filial San Juan de Dios, Estelí}

La Clínica Médica Previsional San Juan de Dios es una institución de que brinda servicios de salud que está adscrita al MINSA garantiza la atención medica diferenciada mediante sus recurso y capacidad instalada a fin de resolver la demanda de atención por problemas de salud y sus complicaciones para los asegurados adscritos a esta clínica. La gerencia de la clínica médica previsional, MINSA/ filial San Juan de Dios, Estelí, tomando en cuenta el crecimiento y desarrollo tecnológico y humano que ha tenido la clínica y consciente de la necesidad de contar con un régimen interno de trabajo, que se ajuste a la situación actual y que sea al mismo tiempo congruente con el marco jurídico establecido en el código del trabajo ha orientado la elaboración del presente reglamento interno de trabajo.

Este reglamento contiene disposiciones de carácter general y disciplinario y constituye la base fundamental que regulara y garantizara que las relaciones laborales 
entre la clínica y sus trabajadores, sean de armonía, justicia, equidad y que se desarrollen en un ambiente agradable lo que conllevara a un funcionamiento adecuado del mismo, así como la optimización de los recursos humanos.

En la actualidad el nivel de resolución que ha alcanzado la Clínica San Juan de Dios implica una serie de procesos necesarios para alcanzar la mejor calidad en pos de la atención del asegurado, lo que significa un incremento en los recursos humanos y técnicos así como también fortalecer el sistema contable de la Clínica para obtener resultados contables confiables, creando un plan de mejoramiento continuo de la calidad que sea medible y alcanzable.

\section{Evaluación del desempeño por el método de escalas graficas con utilización de puntos}

Este método es el más utilizado y divulgado. Aunque, exige múltiples cuidados en su aplicación para evitar la subjetividad y los prejuicios del evaluador que podrían causar interferencias considerables.

\section{"Utilizamos el método de evaluación que generalmente} se utiliza en todas las empresas, el método de escalas graficas por porcentajes de evaluación con respecto al desempeño de cada colaborador" (Lic. Leydi Martínez. Analista RRHH de Clínica Previsional).

El proceso de evaluación del desempeño que se realiza en la clínica médica previsional según Lic. Leydi Martínez analista de recursos humanos se lleva a cabo de la siguiente manera:

- Se orienta a los jefes de las 13 áreas, tenemos trece áreas.

- Les doy un formato para que cada jefe inmediato evalué a cada trabajador, porque son muchos y no puedo evaluar personalmente.

- Remiten el formato de evaluación de trabajador y reviso si está bien la puntuación de cada trabajador.
- Posteriormente se revisan quienes son las personas que tienen las puntuaciones más bajas y los criterios donde están fallando, para implementar con ellos una concientización de mejoras en el desempeño de sus funciones.

"El proceso continuo de proporcionar a los subordinados, información sobre la eficacia con que están efectuando su trabajo para la organización" (Lic. Walter Espinoza jefe inmediato del área de farmacia).

Instrumento de evaluación del personal, utilizado por la clínica médica previsional MINSA filial San Juan de Dios

La clínica médica previsional utiliza el método de evaluación de desempeño por escala gráficas, basado en un manual de gestión de desempeño utilizado por el MINSA a nivel nacional, en el cual de acorde al perfil de cada área de trabajo y de cada servicio en este caso, elaboramos los objetivos a seguir para la evaluación de desempeño y por ello cada área y servicio tiene su objetivo por separado. (Lic. Leydi Martínez / analista de recursos humanos).

Los parámetros son indicadores, datos del resultado deseado en la ejecución de alguna tarea. Los criterios de desempeño están relacionados con las funciones principales del puesto y constituyen no sólo una lista de tareas sino que describen lo que el empleado debe lograr en el desempeño de su puesto.

Los parámetros a considerarse para evaluar el desempeño; son parámetros de control interno entre estos entradas, salidas, porte y aspecto, disciplina laboral, trabajo en equipo, atención al paciente lo cual en esta área para nosotros es lo más importante, flujo de información y sigilo. (Lic. Leydi Martínez / analista de recursos humanos).

Para Lic. Moreno "los parámetros más importantes que se toman en cuenta en la evaluación que nos realizan 
son: Uniforme, responsabilidad, puntualidad, interés por el trabajo compañerismo y las buenas relaciones como compañeros". "esta evaluación si es importante que se hagan por que ayudan, pero aqui de nada sirve hacerlo porque no se ve el cambio todo sigue igual".

El instrumento que se utiliza es muy importante porque es a través de este que se evalúa las funciones de cada trabajador y específicamente en el área de farmacia la atención que se les brinda a los pacientes.

La frecuencia con que se realizan las evaluaciones del desempeño en esta institución es dos veces al año, es decir semestralmente.

\section{Método de evaluación del desempeño que utiliza la clínica médica previsional para evaluar al personal.}

El método de evaluación de escalas graficas o Método de Porcentaje está definido por el Ministerio de Salud (MINSA) para evaluar el desempeño del personal de los trabajadores de los Hospitales y las clínicas médicas previsionales del país.

En este estudio se presenta la aplicación del Método para el caso de la Clínica Previsional MINSA Filial San Juan de Dios de la ciudad de Estelí cuyos criterios de medida para cada objetivo se detallan a continuación:

\section{Disciplina, asistencia y puntualidad}

Record de puntualidad: consiste en una regla estipulada en esta institución la cual los trabajadores deben cumplir, se estipula llegar diez (10) minutos antes de la hora de entrada para cumplir el deber de anotar en el sistema su llegada, el control de la puntualidad se mide en base al registro de entradas y salidas, haciendo uso del reloj de huella en el cual se detalla: Nombre del trabajador, área de trabajo, hora de llegada y hora de salida en los diferentes turnos.

Cumplimiento de horarios: Este criterio se mide de acuerdo a la asistencia a los turnos asignados a los trabajadores y el cumplimiento completos de sus horas. El cumplimiento de este horario se registra utilizando el control de huella.

Interrupciones innecesarias: Este criterio es medido de acuerdo a una encuesta que se realiza una vez al mes, la clínica a sus asegurados para conocer la atención que reciben de parte de los trabajadores en diferentes áreas de la clínica. Algunas de las preguntas que realizan en esta encuesta son: ¿Cuándo usted es atendido/a el despachador/a hace interrupciones?; ¿Qué tipo de interrupciones? (hace comentarios ofensivo, conversa con sus compañeros de trabajo al momento de atenderle, atiende llamadas o mensajes de texto y le deja esperando por mucho tiempo mientras atiende asuntos personales).

Permisos, citas médicas: Este criterio se mide de acuerdo a la cantidad de ausencias que tienen los trabajadores de esta institución. Estas ausencias pueden ser por permisos personales, citas médicas, por emergencia o los permisos pre y postnatal y de paternidad. El jefe de área es quien lleva un registro de los permisos otorgados a sus subordinados los cuales presentan un soporte (constancia o en un cuaderno), para justificar su ausencia. En cuanto a los subsidios (prenatal, posnatal, enfermedades comunes o accidentes laborales) se les llena la esquela de subsidios si el reposo es mayor a 48 horas luego esta esquela el jefe de área la envía al área de recursos humanos y la analista hace un informe mensual que los lleva los 1 de cada mes al Instituto nicaragüense de seguridad social (INSS). En cuanto a las ausencias no justificadas la Clínica permite a cada trabajador un máximo de cuatro ausencias por mes, si excede esta cantidad el trabajador es sancionado por irresponsabilidad.

Cumple reglamento disciplinario: Existe dentro de Normas del Ministerio de Salud el reglamento disciplinario donde se especifican las faltas en las que puede incurrir un trabajador, estas faltas se clasifican en graves, moderadas y leves, las faltas leves se 
corrigen con llamados de atención y memorándum. Las faltas moderadas se corrigen haciendo cartas de compromiso a los trabajadores. Las faltas graves suele ser corregidas con cancelar el contrato al trabajador.

\section{Relaciones interpersonales}

Comunicación con los jefes: Este punto es medido por el jefe de cada área el cual lo valora mediante las relaciones de confianza, ayuda mutua, compañerismo y relaciones interpersonales. Cabe destacar que en este caso la valoración es subjetiva porque no se cuenta con un instrumento de control.

Comunicación y conducta amable con los pacientes: Este criterio al igual que otros también se toma de la opinión del usuario mediante la encuesta que se les aplica y por la observación de las supervisiones de los jefes de área quienes son los encargados de velar que cada trabajador realizar las tareas encomendadas en tiempo y forma.

Evita conflictos: Se mide a través de los jefes de áreas y supervisores los cuales siguen de cerca las relaciones entre compañeros y la atención que brindan al usuario.

Frecuencia de quejas: Este criterio lo miden los usuarios que reciben los diversos servicios que ofrece esta institución los cuales realizan sus quejas a través del uso del buzón de sugerencia que se revisado semanalmente por el director, analista de recursos humanos, sindicalista quienes revisan cada queja para dar respuesta y mejorarla atención del usuario.

\section{Disponibilidad}

Acata órdenes y cumple orientaciones: Este punto consiste en la delegación de órdenes por parte de los jefes inmediatos hacia los subordinados y el cumplimiento de las mismas. Los jefes llevan un registro sobre el comportamiento individual de cada trabajador. El cual lo analiza el analista de recursos humanos para la toma de medidas correctivas, en caso de que haya incumplimiento de las tareas asignadas.

Trabajo extraordinario: consiste en la jornada realizada por un trabajador fuera de su horario estipulado en el contrato de trabajo. Este criterio es medido por los jefes de área en cuanto al cumplimiento de las funciones y tareas asignadas fuera del horario de trabajo, dichas horas extras se dan cuando se realizan inventarios, revisión de medicamentos nuevos los cuales se deben recibir por tipo y garantizar las medidas necesarias para evitar el deterioro de dicho medicamento. Este tiempo es remunerado según lo establece las leyes del código de trabajo.

\section{Trabajo en equipo}

Actúa en conjunto con los/as compañeros/as de trabajo y Coordina con los jefes inmediatos: Este criterio consiste en las relaciones de compañerismo y trabajo en equipo, puede consistir en la organización de festividades de días especiales (cumpleaños, día de la madre, día del padre, día del trabajador de la salud) jornadas de limpieza, control de inventario, campañas de vacunación u otras afectaciones definidas por el Ministerio de Salud. Cada jefe inmediato lleva el control de estas actividades.

\section{a. Niveles de conocimientos y dominio técnico de trabajo}

Domina funciones: El dominio de funciones es la capacidad que posee el trabajador para realizar de manera eficiente y eficaz su trabajo. Este criterio se evalúa en base a las capacitaciones que se le hacen al trabajador y de esta manera se va evaluando si ellos han mejorado sus dificultades y se realiza mensualmente.

Orden y esmero en el trabajo: se valora por el jefe tomando en consideración el desarrollo de funciones, así mismo la iniciativa, integración y motivación para realizar las diversas actividades y cumplir con las tareas estipuladas. 
b. Valoración de los resultados de la evaluación.

Dentro de los resultados obtenidos de la última evaluación realizada a finales de mayo del año 2014 a los trabajadores del área de farmacia, se puede apreciar en el cuadro $\mathrm{N}^{\circ} 1$ para lo cual se consideran los siguientes objetivos: 1) Disciplina, asistencia y puntualidad; 2) Relaciones interpersonales; 3) Disponibilidad; y 4) Trabajo en equipo.

\section{Niveles de conocimiento y dominio técnico de trabajo.}

Así mismo el puntaje es indicado de acuerdo a los niveles de eficiencia:

- Excelente: 91-100\%.

- Muy bueno: 81-90\%.

- Buena: 71-80\%.

- Regular: 61-70\%.

- Insuficiente: 0- 60\%

En este instrumento los principales criterios de medida son:

- Record de puntualidad.

- Cumple horarios.

- Interrupciones necesarias.

- Permisos, citas médicas.

- Cumple reglamento disciplinario.

- Comunicación con los jefes.

- Comunicación con los pacientes.

- Conducta amable.

- Evita conflictos.

- Frecuencia de quejas.

- Acata órdenes.

- Cumple orientaciones.

- Trabajo extraordinario.

- Actúa en conjunto con los/ las compañeras de trabajo.

- Domina funciones.

- Orden y esmero en el trabajo.
Los criterios en los cuales se observa mayor dificultad:

"incidencia más que todo en las entradas y salidas, en otras ocasiones se ponen rebeldes, en el caso de la atención asistencial se traen a veces problemas personales para el área de trabajo, lo que afecta en la atención al paciente y por ende afecta a todos". (Lic. Leydi Martinez, Analista de recursos Humanos).

"una vez identificadas las personas con menores puntajes se procede a llamar a quienes presentaron menor calificación en su evaluación, mediante reuniones reflexivas para lo cual se les da un periodo de tiempo de tres meses para superar estas debilidades, luego se procede a firmar carta de compromiso, si la puntuación alcanza niveles muy bajos se le notifica al trabajador mediante un llamado de atención, si no resulta ninguna acción aplicada se procede al despido, actualmente no se ha llevado a cabo un despido por esta falta”. (Lic. Leydi Martínez, Analista de recursos humanos).

Valoración del desempeño laboral por parte de los usuarios de la clínica médica previsional MINSA filial San Juan de Dios.

\section{Disciplina, asistencia y puntualidad}

Con respecto a la responsabilidad, que como usuarios ven reflejada en el despachador de farmacia un $21.23 \%$ indicó que la atención que han recibido ha cumplido con sus expectativas es decir les han brindado una excelente atención.

Mientras un $10.38 \%$ consideran que el servicio obtenido es bueno, el $61.32 \%$ opinan es regular es decir no fueron mal atendidos, sin embargo no es la atención que esperaban y un $7.07 \%$ indican que la atención es mala es decir que el servicio obtenido no es lo que como usuarios merecen. 
Con respecto a la asistencia en el local de trabajo, encontramos que un $47.17 \%$ de la muestra encuestada indico que en las visitas a la clínica y al momento de presentarse en el área de farmacia, el despachador se encuentra en su lugar de trabajo, un 5.19\% expresa que no han encontrado al trabajador en el período de presentarse a farmacia, el $47.64 \%$ opina que algunas veces han encontrado al despachador de farmacia y otras veces no están en el lugar indicado.

Con respecto a la pregunta acerca de la interrupciones innecesarias que hace el despachador de farmacia al momento de atender al paciente, el $47.64 \%$ de las personas encuestadas considera que mientras es atendido el despachador interrumpe constantemente.

Mientras el $52.36 \%$ de las personas encuestadas afirman que el despachador de farmacia cuando atiende al paciente se dedica a su trabajo y evita hacer interrupciones constantemente.

El servicio al cliente no es una decisión optativa sino un elemento imprescindible para la existencia de una empresa y constituye el centro de interés fundamental y la clave de su éxito o fracaso, en tanto mientras menos interrupciones sean realizadas mayor satisfacción recibe el cliente.

\section{Relaciones interpersonales}

Se le consultó al universo de estudio acerca del grado de confianza que le brindan los despachadores al momento de ser atendidos, un 10.38\% expresa sentir confianza en su despachador para realizar cualquier consulta, licenciada Olivas nos comenta" siempre que he venido a la clínica en farmacia por casualidades de la vida está la misma muchacha, siempre me atiende ella por eso tengo gran confianza con ella”.

Con respecto a la amabilidad con la que atienden en el área de farmacia, encontramos que el 20.28\% de la muestra opina que es excelente, el $47.17 \%$ cree es regular es decir es buena, sin embargo no han recibido la atención con la amabilidad que esperaban y el 32.55\% consideran haber sido atendidos con amabilidad.

Se consultó al universo de estudio acerca del entusiasmo que observan en los trabajadores donde se refleja el $62.26 \%$ su entusiasmo es regular "va en dependencia quien este despachando hay unas que me atienden con amabilidad y si no hay medicamento buscan como resolver mientras hay quienes dicen no hay y no buscan opciones.

\section{Disponibilidad}

Según los datos que resultaron de las encuestas el $33.02 \%$ expresa que si le han explicado en muchas ocasiones el uso correcto del medicamento y el procedimiento para suministrarlo, así mismo un $33.02 \%$ indican durante las visitas realizadas a esta área nadie les explico cómo se debe usar el medicamento brindado "quienes expresan el doctor lo receto el debió explicarle como suministrarlo", el 33.96\% opinó que pocas son las veces que han recibido explicación de su medicamento.

Con respecto a la consulta acerca de la entrega posterior a su medicamento, se encontró que un $46.70 \%$ expresa que si recibe el medicamento una vez que se presenta a farmacia y posterior a su consulta, por el contrario el $53.30 \%$ indica que prefiere comprarlo fuera, debido a que muchas veces hacen fila y cuando llegan a ser atendido no encuentran el medicamento que necesitan.

Con respecto a la pregunta acerca del espacio físico donde está ubicada el área de farmacia el 92.92\% coincide que hay necesidad de un nuevo local, según licenciada Corrales "Yo considero que el espacio del cual disponen es muy pequeño para demanda de usuarios que tiene la clínica."

Con respecto a las ventanillas de atención al usuario en el área de farmacia de la clínica el 77.83 \% considera 
que no son las suficientes para atender a todos los usuarios de este servicio diariamente, mientras el $22.16 \%$ respondió que las ventanillas que existen en atención al usuario son las suficientes para la atención.

Para el 77.83\% de los usuarios con la ampliación de más ventanillas de atención al usuario se hace necesario que se contrate a más personal para atender con más rapidez.

\section{Conocimiento y dominio técnico del trabajo}

Se consultó la opinión del usuario con respecto a la formación profesional que refleja el personal de farmacia, donde se encontró un 57.08\% de acuerdo que es la adecuada, expresando que si no fuese así no estaría ocupando este cargo, mientras el 42.92\% opina que quienes lo han atendido no han demostrado formación profesional, al respecto licenciado Rodríguez expresa "a mi criterio, dominan la teoría pero no lo llevan a la práctica, es decir el conocimiento esta, sin embargo sin temor a equivocarme la atención que nos brindan no es la muchos esperamos".

El $73.58 \%$ de personas encuestadas indican que el despachador de farmacia al momento de entregarle su tratamiento no le dice el procedimiento a seguir para tomarse el medicamento, "si se les pregunta algunos explican detenidamente pero si no se les pregunta no toman iniciativa para explicarlo, asimismo va en dependencia de quien este atendiendo", por lo contrario el $26.42 \%$ indican siempre que recibe este servicio se le ha explicado cómo debe suministrar el medicamento recetado por el médico.

Se consultó a las personas encuestadas acerca del nivel de conocimiento que presenta el despachador de farmacia donde se encuentra un 33.49\% que expresan que su dominio es excelente, un $42.92 \%$ cree que su nivel de dominio es regular es decir conocen, "si están en el cargo es porque realmente dominan y son capaces aunque en ocasionan no lo reflejan", por el contrario el
$23.59 \%$ expresa desde que el dominio presentado por los despachadores no es muy bueno.

\section{CONCLUSIONES}

La Clínica médica previsional MINSA filial San Juan de Dios, presenta muchos recursos para lograr cumplir sus metas, objetivos, misión, visión. La demanda de usuarios del servicio que brinda esta institución está en aumento, sin embargo es necesario que exista mayor y mejor participación del recurso humano para logar cumplir con sus objetivos previstos.

Al determinar el proceso del desempeño laboral que realiza la Clínica médica previsional San Juan de Dios, podemos concluir que se aplica el método de evaluación de escalas graficas con utilización de puntos, el cual se aplica a cada departamento de la clínica, especialmente en el área de farmacia dando a veces resultados muy bajos en las calificaciones y según algunos de los trabajadores de farmacia aunque recursos humanos hace el llamado de atención no se genera ningún cambio.

La demanda de usuarios del servicio que brinda esta institución está en aumento, por lo cual no están en un cien por ciento conforme con la atención que reciben por parte de los despachadores, su inconformidad radica en las interrupciones cuando se les está atendiendo, la mala actitud del despachador al momento de hacer alguna consulta entre otras, por lo cual los usuarios calificaron la atención en un $50.95 \%$ como buena.

Para lograr un mejor rendimiento del desempeño de los trabajadores del área de farmacia se diseñaron una serie de estrategias para que el área de recursos humanos las incorpore y de esta manera promover constantemente las buenas relaciones en el equipo de trabajo, se logre una mejor comunicación y por ende hacer realidad la misión que se ha propuesto la institución. 


\section{RECOMENDACIONES}

Para que esta investigación sea de utilidad al área de recursos humanos de la Clínica médica previsional San Juan de Dios, así como al personal interno del área de farmacia de esta institución y la comunidad estudiantil de la Facultad Regional Multidisciplinaria. Se recomienda:

\section{A la empresa:}

1. Que el sistema de evaluación que se aplica debe estar relacionada más específicamente con el puesto de trabajo.

2. Implementar un proceso de capacitación integral y flexible que permita atender y contribuir a superar las debilidades en dicha área.

3. Que se programe con mayor frecuencia la evaluación del desempeño.

4. Implementar medidas más drásticas con respecto a las sanciones estipuladas en personas reincidentes con esta problemática.

\section{A los trabajadores:}

1. Cumplir adecuadamente con los requisitos propuestos en el manual de funciones de la institución.

2. Que se tome con mayor importancia las funciones de su trabajo y aprovechar las oportunidades de mejora, para mayor estabilidad en el puesto.

\section{BIBLIOGRAFÍA}

Blandón R., Sobalvarro M. (2008). Evaluación del desempeño al personal administrativo de la empresa Barreda Constructora Eléctrica, EMBACONEL S.A. Estelí. Tesis para optar al título de administración de empresas, Facultad Regional Multidisciplinaria, Estelí, Nicaragua.

Colecciones manfut (2014), Nicaragua en cada lugar. (en línea) consultado (25/06/2014). Disponible en http://www.manfut.org/esteli/esteli.html

Midence R., Flores Y. (2005). Desarrollo de recursos humanos en Casa Pellas Estelí. Tesis para optar al título de administración de empresas, Facultad Regional Multidisciplinaria, Estelí, Nicaragua.

Real Academia Española. Diccionario de la Lengua Española - Vigésima Segunda Edición, obtenido en http://buscon.rae.es/draeI/SrvltConsulta?TIPO_ BUS=3\&LEMA=eficiencia, el 26 de abril, 2014.

Rojo, O. (2006). Capacitación y desarrollo de recursos humanos. Consultada el 13 de abril 2014, de http/ www.mailxmail.com/curso capacitación-cursorecursos-humanos/capacitación-curso-recursoshumanos.

Vallejos L., López W (2011). Importancia de la evaluación del desempeño laboral en las fincas de producción de la empresa Tabacos de Oriente de Nicaragua S.A en el municipio de Estelí. Tesis para optar al título de administración de empresas, Facultad Regional Multidisciplinaria, Estelí, Nicaragua.

Web de recursos humanos y el empleo (2014), Proceso de evaluación del desempeño (en línea) consultado (29/06/2014). Disponible en http://www.rrhh-web. com/evaluaciondepuesto.html 